九州大学学術情報リポジトリ

Kyushu University Institutional Repository

\title{
Temperature dependent thermal conductivity of a suspended submicron graphene ribbon
}

\author{
Li, Qin-Yi \\ Key Laboratory for Thermal Science and Power Engineering of Ministry of Education, Department
} of Engineering Mechanics, Tsinghua University

Takahashi, Koji

International Institute for Carbon Neutral Energy Research (WPI-I2CNER), Kyushu University

Ago, Hiroki

Institute for Material Chemistry and Engineering, Kyushu University

Zhang, Xing

Key Laboratory for Thermal Science and Power Engineering of Ministry of Education, Department of Engineering Mechanics, Tsinghua University

他

http://hdl. handle. net/2324/4354937

出版情報: Applied physics letters. 117 (6)，pp.065102-，2015-02-10. AIP Publishing バージョン：

権利関係: (C) 2015 AIP Publishing LLC 


\section{Temperature dependent thermal conductivity of a suspended submicron graphene ribbon}

Cite as: J. Appl. Phys. 117, 065102 (2015); https://doi.org/10.1063/1.4907699

Submitted: 04 January 2015 . Accepted: 24 January 2015 . Published Online: 10 February 2015

Qin-Yi Li, Koji Takahashi, Hiroki Ago, Xing Zhang, Tatsuya Ikuta, Takashi Nishiyama, and Kenji Kawahara
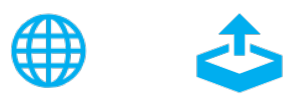

\section{ARTICLES YOU MAY BE INTERESTED IN}

Temperature dependent thermal conductivity of a free-standing graphene nanoribbon Applied Physics Letters 102, 111911 (2013); https://doi.org/10.1063/1.4796177

Extremely high thermal conductivity of graphene: Prospects for thermal management applications in nanoelectronic circuits

Applied Physics Letters 92, 151911 (2008); https://doi.org/10.1063/1.2907977

Nanoscale thermal transport

Journal of Applied Physics 93, 793 (2003); https://doi.org/10.1063/1.1524305

New

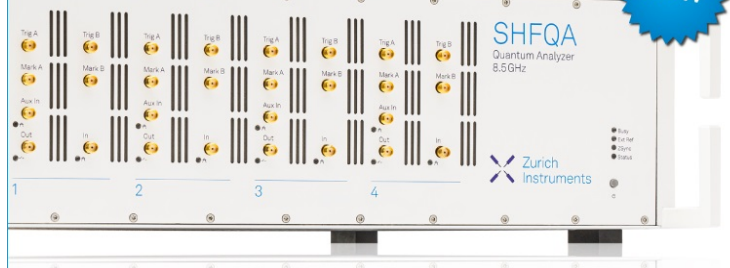

Your Qubits. Measured.

Meet the next generation of quantum analyzers

- Readout for up to 64 qubits

- Operation at up to $8.5 \mathrm{GHz}$, mixer-calibration-free

- Signal optimization with minimal latency
Find out more

Zurich

Instruments 


\title{
Temperature dependent thermal conductivity of a suspended submicron graphene ribbon
}

\author{
Qin-Yi Li, ${ }^{1,2}$ Koji Takahashi, ${ }^{2,3,4}$ Hiroki Ago, ${ }_{5}^{5}$ Xing Zhang, ${ }^{1,2,4, a)}$ Tatsuya Ikuta, ${ }^{3,4}$ \\ Takashi Nishiyama, ${ }^{3,4}$ and Kenji Kawahara ${ }^{5}$ \\ ${ }^{1}$ Key Laboratory for Thermal Science and Power Engineering of Ministry of Education, \\ Department of Engineering Mechanics, Tsinghua University, Beijing 100084, China \\ ${ }^{2}$ International Institute for Carbon Neutral Energy Research (WPI-I2CNER), Kyushu University, \\ 744 Moto-oka, Nishi-ku, Fukuoka 819-0395, Japan \\ ${ }^{3}$ Department of Aeronautics and Astronautics, Kyushu University, Fukuoka 819-0395, Japan \\ ${ }^{4}$ JST, CREST, Kyushu University, Fukuoka 819-0395, Japan \\ ${ }^{5}$ Institute for Material Chemistry and Engineering, Kyushu University, Fukuoka 816-8508, Japan
}

(Received 4 January 2015; accepted 24 January 2015; published online 10 February 2015)

\begin{abstract}
Thermophysical characterization of graphene is very important for both fundamental and technological research. While most of the existing thermal conductivity measurements are for graphene sheets with sizes larger than $1 \mu \mathrm{m}$, the thermal conductivities for suspended submicron graphene ribbons are still very few, although the thermal conductivity of graphene ribbons at the submicron scale is predicted to be much smaller than large graphene and strongly size dependent for both length and width due to the $2 \mathrm{D}$ nature of phonon transport. Here, we report the temperature dependent thermal conductivity of a 169-nm wide and 846-nm long graphene ribbon measured by the electrical self-heating method. The measured thermal conductivities range from $(12.7 \pm 2.95) \mathrm{W} / \mathrm{m} / \mathrm{K}$ at $80 \mathrm{~K}$ to $(932 \pm 333) \mathrm{W} / \mathrm{m} / \mathrm{K}$ at $380 \mathrm{~K}$, being $(349 \pm 63) \mathrm{W} / \mathrm{m} / \mathrm{K}$ at $300 \mathrm{~K}$, following $\mathrm{a} \sim T^{2.79}$ law for the full temperature range of $80 \mathrm{~K}$ to $380 \mathrm{~K}$ and $\mathrm{a} \sim T^{1.23}$ law at low temperatures. The comparison of the measured thermal conductance with the ballistic transport limit indicates diffusive transport in this narrow and short ribbon due to phonon-edge as well as phonon-defect scattering. The data were also combined with an empirical model to predict possible width dependence of thermal conductivity for suspended graphene ribbons. These results help understand the 2D phonon transport in suspended submicron graphene ribbons and provide knowledge for controlling thermophysical properties of suspended graphene nanoribbons through size manipulation. (C) 2015 AIP Publishing LLC. [http://dx.doi.org/10.1063/1.4907699]
\end{abstract}

\section{INTRODUCTION}

As a 2D material with superior thermal conductivity, graphene is regarded as a good candidate for thermal management in nano devices and its thermophysical characterization is very important for both fundamental research and technological applications. ${ }^{1}$ The first few measured thermal conductivities of graphene were obtained by Balandin et al. ${ }^{2,3}$ using a Raman spectroscopy technique and the $\sim 10 \mu \mathrm{m}$ long suspended exfoliated graphene samples were measured to have thermal conductivities of $4000-5300 \mathrm{~W} / \mathrm{m} / \mathrm{K}$, superior to both diamond and carbon nanotubes. However, the following several Raman based measurements ${ }^{4-7}$ showed much smaller thermal conductivities for micro size suspended graphene samples, ranging from $600 \mathrm{~W} / \mathrm{m} / \mathrm{K}$ to about $2000 \mathrm{~W} / \mathrm{m} / \mathrm{K}$ at room temperature. The large discrepancy of Raman based data mainly resulted from the low sensitivity of Raman signals with respect to temperature as well as the estimation of laser absorption. ${ }^{8}$ In the Raman based measurements, the temperature rise at the laser spot should be $\sim 50 \mathrm{~K}$ to acquire enough temperature accuracy and thus the measured temperature dependent thermal conductivities also had large error

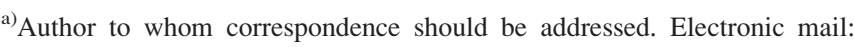
x-zhang@tsinghua.edu.cn. Tel./Fax: 86-10-62772668.
}

bars in the temperature axis. ${ }^{5}$ For more accurate electricalheating based measurement, Seol et al. ${ }^{9}$ fabricated suspended beams of graphene $/ \mathrm{SiO}_{2}$ (1.5-3.2 $\mu \mathrm{m}$ wide, 9.5-12.5 $\mu \mathrm{m}$ long) on a $\mathrm{Si}$ wafer coated with $\mathrm{Au}$ films and obtained the thermal conductivity of supported graphene to be about $600 \mathrm{~W} / \mathrm{m} / \mathrm{K}$ at room temperature, with strong interfacial phonon scattering between graphene and $\mathrm{SiO}_{2}$. Several groups ${ }^{10-13}$ successfully integrated graphene with prepatterned SiN/Pt membranes and measured the thermal conductivity of both supported and suspended graphene sheets using the thermal bridge method. Besides, Xie et al. ${ }^{14}$ employed the four-wire electrical selfheating method to measure the thermal conductivity of a large graphene sheet with $385 \mu \mathrm{m}$ width and $508 \mu \mathrm{m}$ length based on the almost linear relationship of graphene's electrical resistance and temperature at 200 to $380 \mathrm{~K}$.

On the other hand, most of the measured graphene samples were several micrometers in size, while the thermal conductivity data of submicron graphene ribbons are still limited, especially for suspended samples, probably due to the fabrication difficulty of suspending this atom thick layer. The intrinsic mean free path of graphene is about $500 \mathrm{~nm}$ for suspended graphene and $100 \mathrm{~nm}$ for $\mathrm{SiO}_{2}$ supported graphene $^{15}$ and thus the thermal conductivity is strongly size dependent for submicron graphene ribbons. The phonon transport process will be quasi-ballistic when the length of a 
graphene ribbon is shortened to be comparable with the intrinsic mean free path while the width is large enough. ${ }^{11,15}$ When the width is narrowed down to be comparable with the intrinsic mean free path, the phonon scattering with edge roughness begins to dominate the phonon transport process and the transport process will be diffusive with the free path shortened by the edges. ${ }^{15}$ An elastic-shell-based calculation $^{16}$ showed that the ballistic thermal conductance per unit cross section, which does not depend on the length, varies with the width for graphene ribbons narrower than $500 \mathrm{~nm}$ but becomes independent of width for ribbons wider than $500 \mathrm{~nm}$. Bae et al. ${ }^{15}$ measured the thermal conductivities of $\mathrm{SiO}_{2}$ supported graphene ribbons with $260 \mathrm{~nm}$ length and $45 \mathrm{~nm}, 65 \mathrm{~nm}, 85 \mathrm{~nm}$, and $130 \mathrm{~nm}$ widths using a metal heater and sensor and the thermal conductivity scaled with width approximately as $\sim W^{1.8 \pm 0.3}$, being about $250 \mathrm{~W} / \mathrm{m} / \mathrm{K}$ for the $130 \mathrm{~nm}$ wide ribbon at $300 \mathrm{~K}$. Xu et al. ${ }^{11}$ measured the length-dependent thermal conductivity of suspended monolayer graphene ribbons with a fixed width of $1.5 \mu \mathrm{m}$ and lengths ranging from $300 \mathrm{~nm}$ to $9 \mu \mathrm{m}$ and obtained thermal conductivities logarithmically divergent with the sample lengths even for graphene ribbons much longer than the intrinsic phonon mean free path. $\mathrm{Xu}$ and co-workers ${ }^{11}$ also provided temperature dependent thermal conductivities of a graphene ribbon with $4 \mu \mathrm{m}$ width and $2.5 \mu \mathrm{m}$ length and demonstrated that the thermal conductivity was weakly width dependent when the width was larger than $1.5 \mu \mathrm{m}$. Xu and co-workers ${ }^{11}$ also suggested that the suspended ribbons could be more strongly width dependent than supported samples. However, the thermal conductivity data for suspended submicron graphene ribbons are still very few, although the thermal conductivity is predicted to be strongly dependent with both length and width at sub-micrometer scale for suspended ribbons due to the $2 \mathrm{D}$ nature of heat flow in graphene.

Here, we report the temperature dependent thermal conductivity of a suspended graphene ribbon with $169 \mathrm{~nm}$ width and $846 \mathrm{~nm}$ length measured by the four-wire electrical self-heating method ${ }^{14,17,18}$ based on the temperature dependent electrical resistance at $80 \mathrm{~K}$ to $380 \mathrm{~K}$. This work will help understand the size dependent phonon transport at sub-micrometer scale.

\section{EXPERIMENT}

\section{A. Sample fabrication}

The four-wire electrical self-heating method was used to measure the thermal conductivity of graphene and the electrical current was passed through the suspended graphene ribbon via $\mathrm{Au} / \mathrm{Cr}$ electrodes deposited onto the graphene ribbon. The main fabrication processes are shown in Fig. 1. First, a large-area graphene sheet grown by $\mathrm{Cu}$-based chemical vapor deposition (Cu-CVD) was transferred to a $10 \times 10 \mathrm{~mm} \mathrm{Si} / \mathrm{SiO}_{2}$ wafer with the help of PMMA. The details of the graphene growth and transfer were described elsewhere. ${ }^{19,20}$ Then, the graphene sheet was patterned into a $500 \mu \mathrm{m}$ long, $\sim 200 \mathrm{~nm}$ wide ribbon via electron beam lithography followed by $\mathrm{O}_{2}$ plasma etching. In a second e-beam lithography step, $8 \mathrm{~nm} \mathrm{Cr}$ and $30 \mathrm{~nm}$ Au patterns were physical vapor deposited onto the $\mathrm{Si}$ wafer as four electrodes, clamping the graphene nanoribbon and ensuring good electrical and thermal contact. Finally, the graphene ribbon was suspended by etching $200 \mathrm{~nm}$ thick $\mathrm{SiO}_{2}$ via buffered hydrofluoric acid (BHF) and dried using a $\mathrm{CO}_{2}$ critical point dryer to avoid damage from surface tension.

Figs. 2(a) and 2(b), respectively, show the top view and tilt $60^{\circ}$ view SEM images of the suspended graphene ribbon. The suspended part was $169 \mathrm{~nm}$ wide and $846 \mathrm{~nm}$ long and the thickness was taken as $0.335 \mathrm{~nm} .{ }^{16}$ Figs. 3(a) and 3(b), respectively, show the Raman spectra and band intensity ratio of the graphene sample. In Fig. 3(a), Raman spectra (1)-(4), respectively, show the spectra after the large-area graphene was transferred onto the $\mathrm{Si} / \mathrm{SiO}_{2}$ wafer (1), after $\mathrm{O}_{2}$ plasma patterning (2), right after ribbon suspending by $\mathrm{BHF}$ etching and critical point dry (3), and after SEM observation (4). Before $\mathrm{O}_{2}$ plasma etching, the Raman spectrum showed

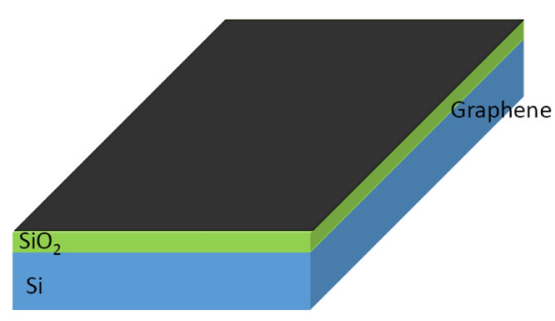

Cu-CVD graphene transferred to Si wafer

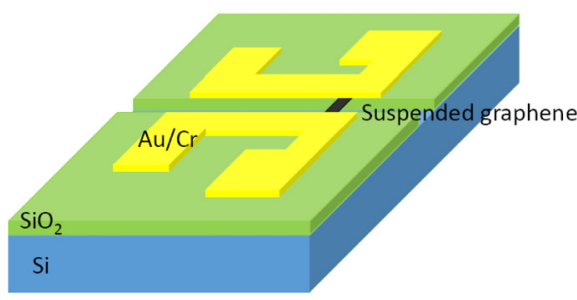

$\mathrm{SiO}_{2}$ etched by $\mathrm{BHF}+$ critical point dry: suspending ribbon

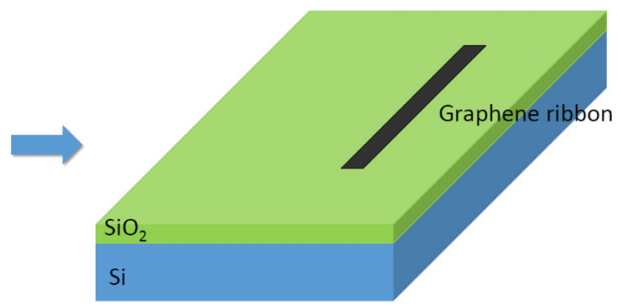

$\mathrm{EBL}+\mathrm{O}_{2}$ plasma: patterning graphene nanoribbon
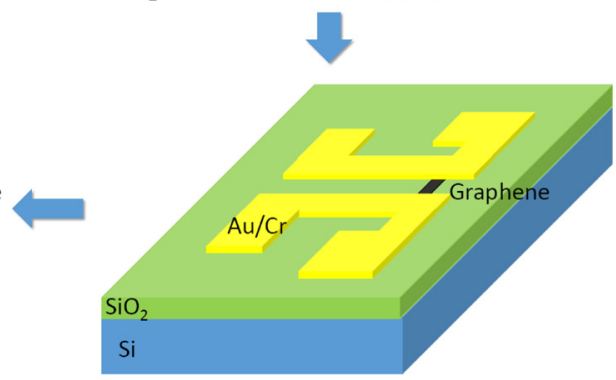

$\mathrm{EBL}+\mathrm{PVD}: \mathrm{Au} / \mathrm{Cr}$ electrodes
FIG. 1. Main fabrication process of the suspended graphene nanoribbon with 4 $\mathrm{Au} / \mathrm{Cr}$ electrodes. 


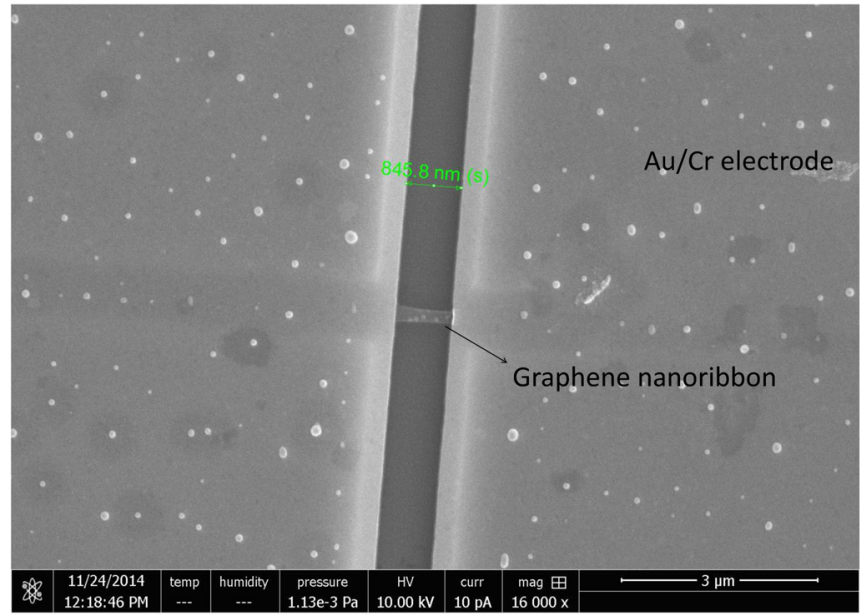

(a)

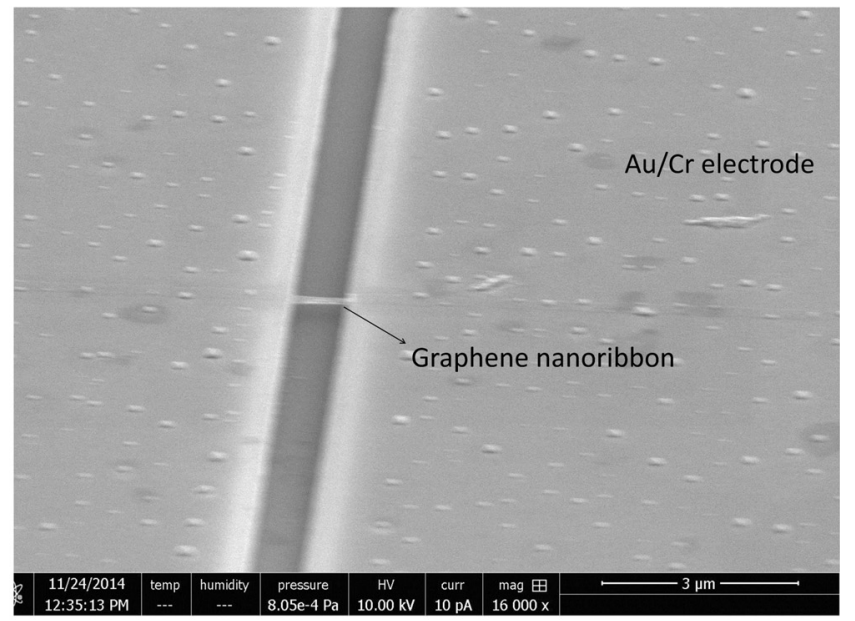

(b)

FIG. 2. SEM images of the suspended graphene ribbon $(169 \times 846 \mathrm{~nm})$. (a) Top view; (b) tilt $60^{\circ}$ view.

no $\mathrm{D}$ band (no defects) and the 2D band intensity was almost twice of $\mathrm{G}$ band, indicating high quality of the monolayer graphene. ${ }^{15}$ After $\mathrm{O}_{2}$ plasma patterning, the Raman spectrum of Fig. 3(a) (2) showed notable D band and the intensity ratio of the $\mathrm{D}$ band to the $\mathrm{G}$ band, $I_{\mathrm{D}} / I_{\mathrm{G}}$, was 0.3 , indicating the effects of edge disorder for this narrow ribbon. ${ }^{15}$ Actually, $I_{\mathrm{D}} / I_{\mathrm{G}}$ varies with the width of graphene ribbons, as shown in Fig. 3(b), where the square symbols are the reference data ${ }^{15}$ for $260 \mathrm{~nm}$ long ribbons and the sphere symbol is the present data from Fig. 3(a) (2), indicating increasing influence of the edge disorder as the graphene ribbon is narrowed. Right after the graphene ribbon was suspended by BHF etching and critical point dry, the Raman spectrum of Fig. 3(a) (3) showed some noise caused by the residue from the wet etching and drying processes and tilt baseline caused by the laser reflection from the adjacent metal electrodes, while the intensity ratio, $I_{\mathrm{D}} / I_{\mathrm{G}}$, was 0.5 . After SEM observation for confirming the ribbon suspension, the $\mathrm{D}$ band was significantly enhanced with $I_{\mathrm{D}} / I_{\mathrm{G}}$ becoming 1.0 , indicating the graphene ribbon was defected by the electron beam. However, the graphene quality after SEM observation was still better than the $130 \mathrm{~nm}$ wide ribbon as shown in Fig. 3(b).

\section{B. Measurement method}

The suspended graphene nanoribbon was heated by direct current and the average temperature rise can be detected through the electrical resistance based on the temperature-resistance relationship of the graphene nanoribbon. The thermal conductivity of the nanoribbon, $\lambda$, can be extracted from the one dimensional heat conduction equation and can be expressed as Eq. (1) ${ }^{17,18}$

$$
\lambda=\frac{R^{\prime} l}{12 w h} \frac{\mathrm{d} P}{\mathrm{~d} R},
$$

where $l$ is the nanoribbon length, $w$ is the nanoribbon width, $h$ is the nanoribbon thickness $(0.34 \mathrm{~nm}) ; R$ is the electrical resistance, $P$ is the electrical power, $\mathrm{d} P / \mathrm{d} R$ is the slope of the $P-R$ curve; $R^{\prime}=\mathrm{d} R / \mathrm{d} T$ is the slope of the calibrated $R-T$ curve at the ambient temperature.
During the measurement, $0.2-25 \mu \mathrm{A}$ direct currents (Power supplier: Advantest R6243) were passed through the suspended graphene nanoribbon via $\mathrm{Au} / \mathrm{Cr}$ electrodes in a vacuum chamber (Oxford Instruments Optistat CF-V-KT). Pressures were lower than $5 \times 10^{-4} \mathrm{~Pa}$ and the heat loss from the nanoribbon to the environment could be neglected. The graphene nanoribbon was connected to a $1 \mathrm{k} \Omega$ standard resistor (Yokogawa 2792 Standard Resistor) and the electrical current was measured from the voltage of the standard resistor. The voltages of the graphene nanoribbon and the standard resistor were measured by 8.5-digit Keithley 2002 Multimeters. As for the thermal contact resistance at the $\mathrm{Au} /$ $\mathrm{Cr} /$ graphene/ $\mathrm{SiO}_{2}$ interfaces, several measurements ${ }^{7,21,22}$ showed that the thermal interfacial resistance is on the order of $10^{-8} \mathrm{~m}^{2} \mathrm{~K} / \mathrm{W}$. In our device, the contact part at each electrode is a long ribbon of $\sim 250 \mu \mathrm{m}$ length and $\sim 200 \mathrm{~nm}$ width. Assuming that the effective contact area is $100 \mu \mathrm{m} \times 200 \mathrm{~nm}=20 \mu \mathrm{m}^{2}$ and that the thermal interfacial resistance is $10^{-7} \mathrm{~m}^{2} \mathrm{~K} / \mathrm{W}$, the total thermal contact resistance will be $1 \times 10^{4} \mathrm{~K} / \mathrm{W}$. Taking the thermal conductivity of the suspended graphene ribbon as $1000 \mathrm{~W} / \mathrm{m} / \mathrm{K}$, the thermal resistance of the suspended ribbon will be $1.5 \times 10^{7} \mathrm{~K} /$ $\mathrm{W}$. The thermal contact resistance is $0.07 \%$ of the suspended ribbon's thermal resistance and thus can be neglected.

\section{RESULTS AND DISCUSSION}

Figs. 4 and 5, respectively, show the temperature dependent electrical resistance and electrical conductivity of the suspended graphene nanoribbon at $80 \mathrm{~K}$ to $380 \mathrm{~K}$. Low currents of $0.2-2 \mu \mathrm{A}$ were used to determine the electrical resistance and the error bars in Figs. 4 and 5 indicate the data dispersion at low currents. The electrical conductivity increases from $8.2 \times 10^{8}$ to $9 \times 10^{8} \Omega^{-1} \mathrm{~m}^{-1}$, which is on the same order as large graphene sheets, ${ }^{14}$ as the temperature rises from $80 \mathrm{~K}$ to $380 \mathrm{~K}$. The electrical resistance does not linearly change with temperature at the full temperature range but can be regarded locally linear at the specific temperature. Especially at $260 \mathrm{~K}$ to $360 \mathrm{~K}$, the electrical 

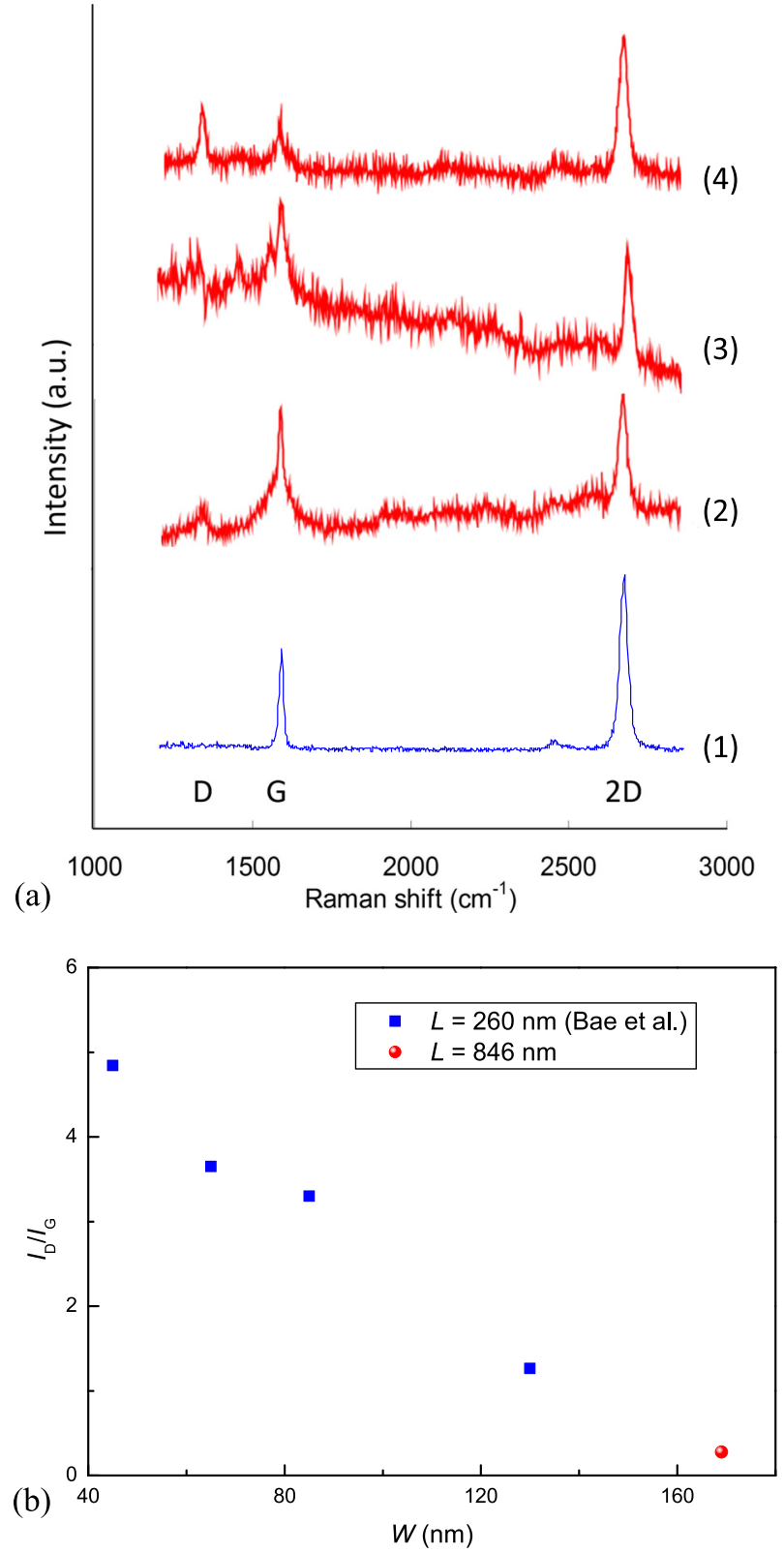

FIG. 3. Raman spectra and band intensity ratio of the graphene sample. (a) Raman spectra of the graphene sample during the fabrication processes: (1) after the large-area graphene was transferred onto the $\mathrm{Si} / \mathrm{SiO}_{2}$ wafer; (2) after $\mathrm{O}_{2}$ plasma patterning; (3) right after suspending by BHF etching and critical point dry; (4) after SEM observation. (b) Intensity ratio of the D band and the $\mathrm{G}$ band varying with the width after $\mathrm{O}_{2}$ plasma patterning, where the square symbols are taken from Ref. 15 for $260 \mathrm{~nm}$ long ribbons and the sphere symbol is the present data from Fig. 3(a) (2).

resistance changes almost linearly with temperature and the $R$ - $T$ slope is $-2.612 \Omega / \mathrm{m}$, as shown in the inset of Fig. 4 .

The local slope of the $R-T$ curve, $R^{\prime}(=\mathrm{d} R / \mathrm{d} T)$, can be approximated by linear fitting the adjacent data at the specific temperature, as shown in Fig. 6. Fig. 7 shows the slope of the $P-R$ curve, $\mathrm{d} P / \mathrm{d} R$, changing with temperature and the inset shows some representative $R$ - $P$ curves at 80 to $380 \mathrm{~K}$ when 3-25 $\mu \mathrm{A}$ currents were passed through the suspended ribbon and the electrical powers were within $10 \mu \mathrm{W}$. As can be seen from the inset of Fig. 7, the R-P curves were not linear for the full power range but sectionally linear, indicating nonlinear $R$-T relationship. For the calculation of $\mathrm{d} P / \mathrm{d} R$,

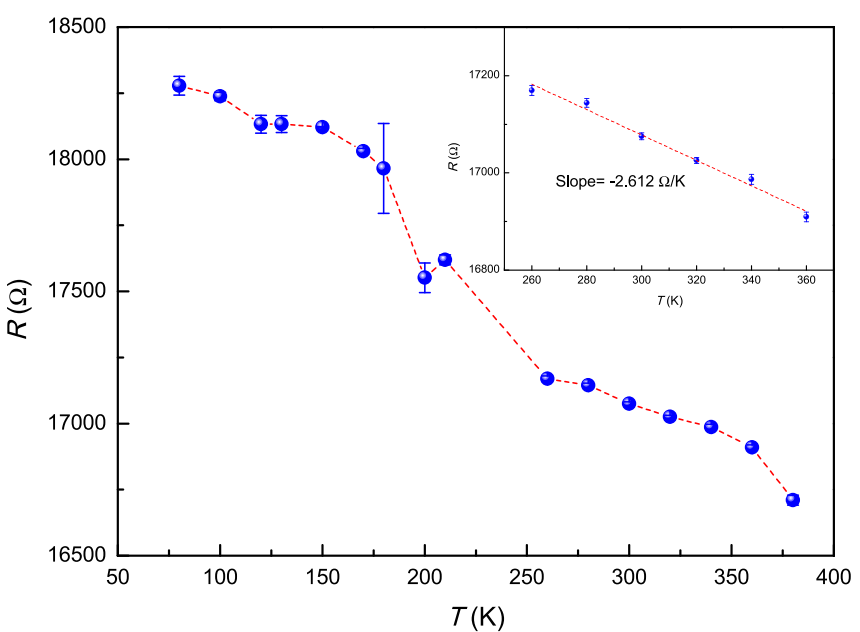

FIG. 4. Temperature dependent electrical resistance of the suspended graphene nanoribbon. Inset: Linear fitting between $260 \mathrm{~K}$ and $360 \mathrm{~K}$.

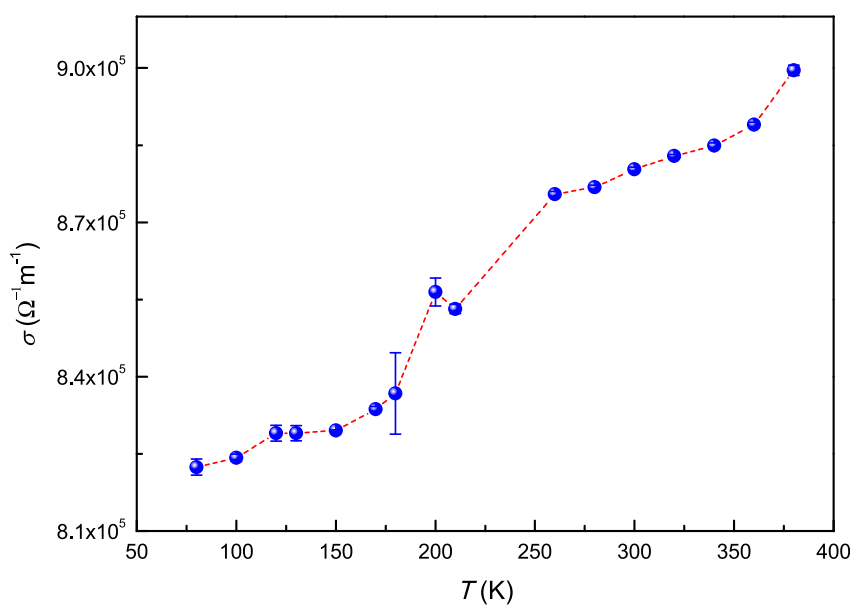

FIG. 5. Temperature dependent electrical conductivity of the suspended graphene nanoribbon.

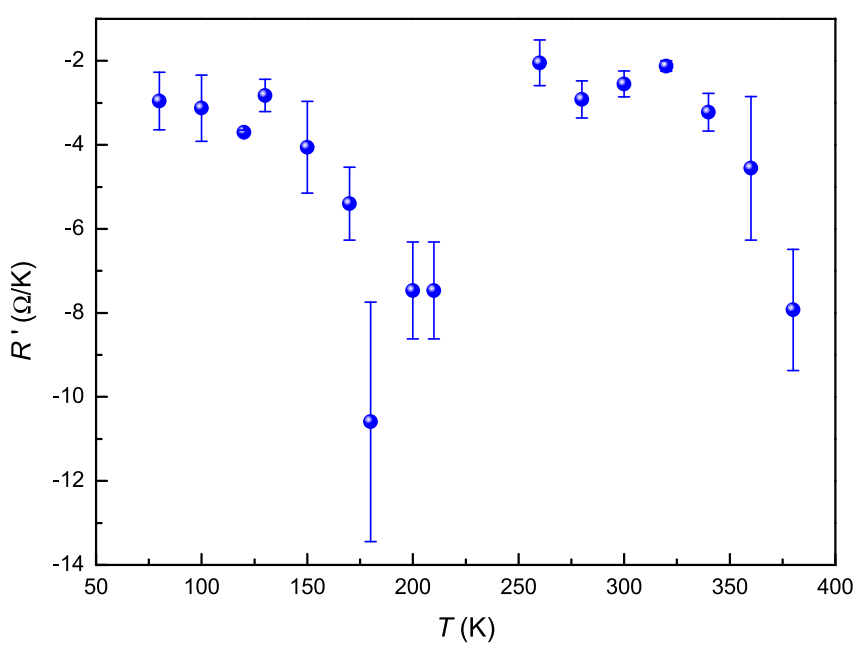

FIG. 6. Temperature dependent $R^{\prime}(=\mathrm{d} R / \mathrm{d} T)$ of the suspended graphene nanoribbon. 


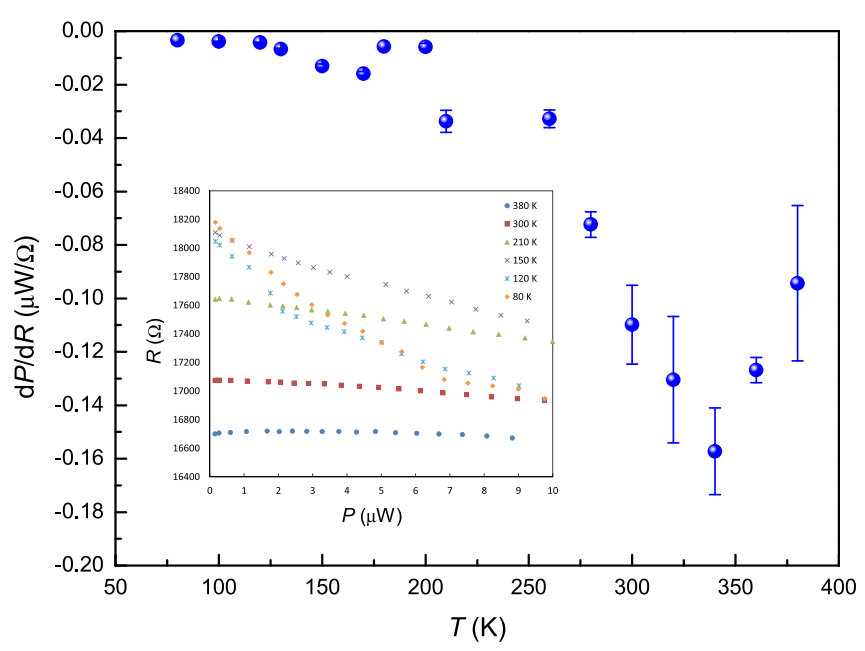

FIG. 7. Temperature dependent $\mathrm{d} P / \mathrm{d} R$ of the suspended graphene nanoribbon. Inset: representative $R-P$ curves at $380 \mathrm{~K}, 300 \mathrm{~K}, 210 \mathrm{~K}, 150 \mathrm{~K}, 120 \mathrm{~K}$, and $80 \mathrm{~K}$ with currents from 3 to $\sim 25 \mu \mathrm{A}$.

only the first 5 to 10 data were used to fit the linear slope, ensuring that the maximum temperature rises were within $10 \mathrm{~K}$ and the error bars in Fig. 7 show the dispersion of the linear fitting results.

Combining $R^{\prime}, \mathrm{d} P / \mathrm{d} R$ and the dimensions of the suspended ribbon, we can calculate the thermal conductivity of the suspended nanoribbon using Eq. (1) and Fig. 8 shows the temperature dependent thermal conductivity with the inset showing the low temperature results. The thermal conductivities can be fit to $\mathrm{a} \sim T^{2.79}$ relationship at the full temperature range of 80 to $380 \mathrm{~K}$, while at low temperatures of 80 to $130 \mathrm{~K}$, the thermal conductivities can be fit to $\mathrm{a} \sim T^{1.23}$ relationship, being $(349 \pm 63) \mathrm{W} / \mathrm{m} / \mathrm{K}$ at $300 \mathrm{~K},(932 \pm 333)$ $\mathrm{W} / \mathrm{m} / \mathrm{K}$ at $380 \mathrm{~K}$, and $(12.7 \pm 2.95) \mathrm{W} / \mathrm{m} / \mathrm{K}$ at $80 \mathrm{~K}$. The present data are much smaller than the thermal conductivity of large graphene sheets, which is about $2000 \mathrm{~W} / \mathrm{m} / \mathrm{K}$ at room temperature, but consistent with the reference data for a $\mathrm{SiO}_{2}$-supported ribbon with $130 \mathrm{~nm}$ width and $260 \mathrm{~nm}$ length $(\sim 250 \mathrm{~W} / \mathrm{m} / \mathrm{K} \text { at } 300 \mathrm{~K})^{15}$ and a suspended ribbon with $3 \mu \mathrm{m}$ width and $500 \mathrm{~nm}$ length $(190 \mathrm{~W} / \mathrm{m} / \mathrm{K}$ at $280 \mathrm{~K}) .{ }^{10}$

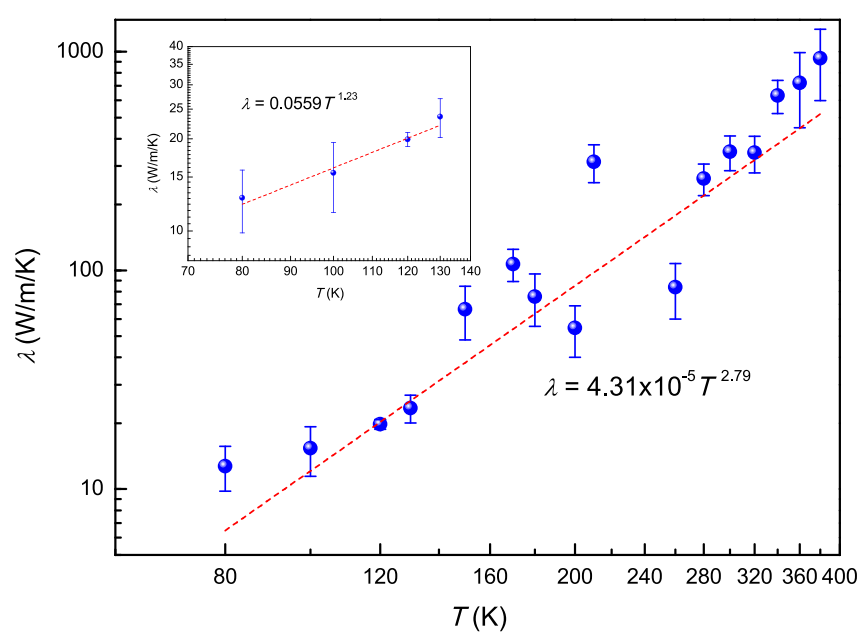

FIG. 8. Temperature dependent thermal conductivity of the suspended graphene nanoribbon, fit to $\mathrm{a} \sim T^{2.79}$ relationship at $80 \mathrm{~K}$ to $380 \mathrm{~K}$. Inset: low temperature $80-130 \mathrm{~K}$ data can be fitted to a $\sim T^{1.23}$ relationship.
Besides, theoretical work ${ }^{16,23}$ has predicted that the low temperature thermal conductivity of graphene follows a $\sim T^{\alpha}$ law, where $\alpha$ varies from 1 for a nanoribbon of $\sim 4 \mathrm{~nm}$ width to 1.5 for graphene sheets with width larger than $500 \mathrm{~nm}$. Xu et al. ${ }^{10}$ also obtained $\mathrm{a} \sim T^{1.53}$ law for a suspended $3-\mu \mathrm{m}$ wide ribbon at temperatures below $150 \mathrm{~K}$. Our present $\sim T^{1.23}$ law for this 169 -nm wide ribbon at low temperatures is in accordance with the theoretical prediction for graphene ribbons narrower than $500 \mathrm{~nm}$.

If the graphene sample is wide and short, which means the length is submicron while the width is larger than $1 \mu \mathrm{m}$, the phonon transport process will be quasi-ballistic and the thermal conductance per unit cross section, $G / A$, will be more instructive than the thermal conductivity. ${ }^{10,11,15,16,21}$ Theoretical work ${ }^{15}$ showed that the simulated ballistic thermal conductance per unit cross section of clean graphene, $G_{\text {ball }} / A$, can be fitted as

$G_{\text {ball }} / A=\left[1 /\left(4.4 \times 10^{5} T^{1.68}\right)+1 /\left(1.2 \times 10^{10}\right)\right]^{-1} \mathrm{WK}^{-1} \mathrm{~m}^{-2}$,

when the temperature ranges from $1 \mathrm{~K}$ to $1000 \mathrm{~K}$. Fig. 9 shows the thermal conductance per unit cross section, $G / A$, of the present short and narrow suspended ribbon and the magenta line indicates the ballistic limit. The measured thermal conductance is $2.3 \%$ of the predicted ballistic limit at $80 \mathrm{~K}$ and $21 \%$ at $380 \mathrm{~K}$. While the reference thermal conductance for wide and short graphene samples was $30 \%-40 \%$ of the ballistic limit, ${ }^{10,11,15}$ our measured data show deviation from quasi-ballistic transport, indicating the fact that the transport in this narrow and short ribbon is indeed diffusive due to phonon-edge scattering. Besides, the sample quality was reduced to some degree during the SEM observation process, as shown in the Raman spectra, which also contributed to phonon-defect scattering and caused deviation from ballistic transport.

The present data can also be extrapolated to predict possible width dependence of thermal conductivity for suspended nanoribbons using a simple empirical model ${ }^{11,15}$ expressed by Eq. (3)

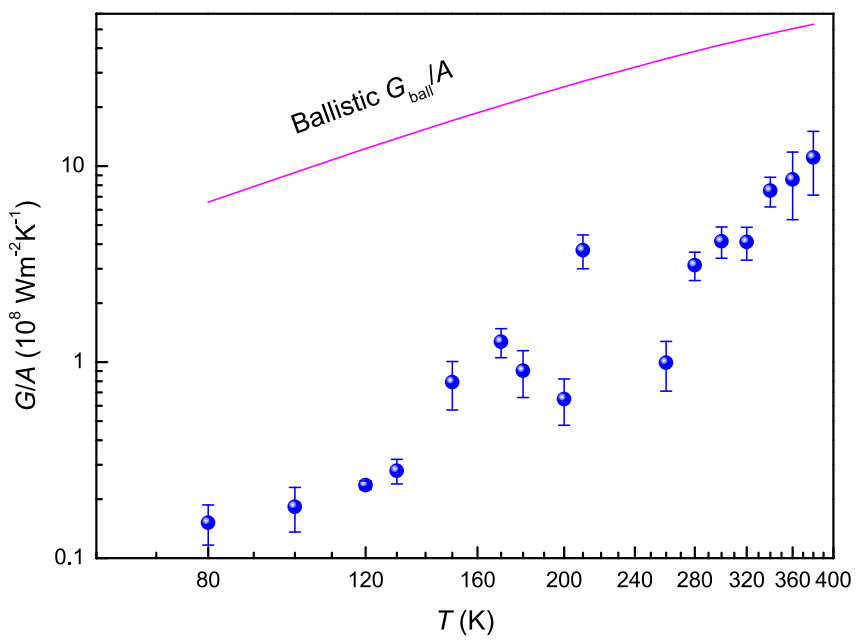

FIG. 9. Temperature dependent thermal conductance per unit cross section, $G / A$. The magenta line indicates the ballistic limit of wide graphene sheets. ${ }^{15}$ 


$$
\lambda_{\mathrm{eff}}(W, L)=\left[\frac{1}{c}\left(\frac{\Delta}{W}\right)^{n}+\frac{1}{\lambda(L)}\right]^{-1},
$$

where $\lambda_{\text {eff }}(W, L)$ is the effective thermal conductivity of a graphene ribbon with width $W$ and length $L ; \Delta$ is the rootmean-square edge roughness, $0.6 \mathrm{~nm} ;{ }^{11,15} c$ is a parameter equaling $0.04 \mathrm{~W} / \mathrm{m} / \mathrm{K}$ at $300 \mathrm{~K} ;{ }^{11,15} n$ is a fitting exponent and $\lambda(L)$ is the length dependent thermal conductivity for quasi-ballistic transport of wide and short graphene ribbons, which is calculated by a simple model ${ }^{15}$ expressed by Eq. (4)

$$
\lambda(L)=\frac{G_{\text {ball }}}{A}\left[\frac{1}{L}+\frac{1}{(\pi / 2) l_{\mathrm{mfp}}}\right]^{-1},
$$

where $G_{\text {ball }} / A$ is the ballistic thermal conductance per unit cross section calculated by Eq. (2) and $l_{\mathrm{mfp}}$ is the intrinsic phonon mean free path of large graphene. To obtain the intrinsic phonon mean free path of suspended graphene, we extracted the only two reference data for width dependent thermal conductivity of suspended ribbons $(W=1.5 \mu \mathrm{m}$, $4 \mu \mathrm{m} ; L=2.5 \mu \mathrm{m})$ from Xu et al. ${ }^{11}$ and reproduced the best fitting using the above empirical model. The reproduced fitting gave the intrinsic mean free path as $214 \mathrm{~nm}$ at $300 \mathrm{~K}$, indicating a thermal conductivity of about $1420 \mathrm{~W} / \mathrm{m} / \mathrm{K}$ for large-area suspended graphene sheets, while the intrinsic mean free path is $90 \mathrm{~nm}$ for $\mathrm{SiO}_{2}$-supported graphene at $300 \mathrm{~K}^{15}$ Using our present data at $300 \mathrm{~K}$ and the intrinsic mean free path of $214 \mathrm{~nm}$, we can obtain the only fitting parameter $n$ as 1.68 for $846 \mathrm{~nm}$ long suspended samples, predicting that a wide $(W>5 \mu \mathrm{m})$ and $846 \mathrm{~nm}$ long ribbon would have a thermal conductivity of about $1000 \mathrm{~W} / \mathrm{m} / \mathrm{K}$ and that a ribbon narrower than $50 \mathrm{~nm}$ would have a thermal conductivity less than $100 \mathrm{~W} / \mathrm{m} / \mathrm{K}$. However, since the available data are very few, the present prediction for width dependent thermal conductivity needs to be validated by systematic measurements for various sizes in our future work.

\section{CONCLUSION}

In conclusion, the existing thermal conductivity data for suspended submicron graphene ribbons are still very few although the thermal conductivity of graphene with submicron sizes is expected to be much smaller than large graphene sheets and strongly size dependent. We measured the temperature dependent thermal conductivity of a suspended graphene ribbon with $169 \mathrm{~nm}$ width and $846 \mathrm{~nm}$ length using the electrical self-heating method based on the relationship of graphene's electrical resistance and temperature. The measured thermal conductivities range from $(12.7 \pm 2.95)$ $\mathrm{W} / \mathrm{m} / \mathrm{K}$ at $80 \mathrm{~K}$ to $(932 \pm 333) \mathrm{W} / \mathrm{m} / \mathrm{K}$ at $380 \mathrm{~K}$, following $\mathrm{a} \sim T^{2.79}$ for the full temperature range of $80 \mathrm{~K}$ to $380 \mathrm{~K}$ and $\mathrm{a} \sim T^{1.23}$ law at low temperatures of $80 \mathrm{~K}$ to $130 \mathrm{~K}$. The thermal conductance per unit cross section is only $2.3 \%$ of the predicted ballistic limit at $80 \mathrm{~K}$, indicating diffusive phonon transport in this submicron ribbon due to phonon-edge and phonon-defect scattering. Moreover, the present data at $300 \mathrm{~K},(349 \pm 63) \mathrm{W} / \mathrm{m} / \mathrm{K}$, were combined with an empirical model to predict possible width dependence of thermal conductivity for 846-nm long suspended graphene ribbons, showing that the thermal conductivity would scale with the width approximately as $\sim W^{1.7}$.

\section{ACKNOWLEDGMENTS}

This work was supported by the National Natural Science Foundation of China (Grant Nos. 51327001, 51356001, 51136001, and 51321002) and the JSPS KAKENHI Grant No. 25289041.

${ }^{1}$ E. Pop, V. Varshney, and A. K. Roy, "Thermal properties of graphene: Fundamentals and applications," MRS Bull. 37, 1273-1281 (2012).

${ }^{2}$ A. A. Balandin, S. Ghosh, W. Bao et al., "Superior thermal conductivity of single-layer graphene," Nano Lett. 8(3), 902-907 (2008).

${ }^{3}$ S. Ghosh, I. Calizo, D. Teweldebrhan, E. P. Pokatilov, D. L. Nika, A. A. Balandin, W. Bao, F. Miao, and C. N. Lau, "Extremely high thermal conductivity of graphene: Prospects for thermal management applications in nanoelectronic circuits," Appl. Phys. Lett. 92, 151911 (2008).

${ }^{4} \mathrm{C}$. Faugeras et al., "Thermal conductivity of graphene in corbino membrane geometry," ACS Nano 4, 1889 (2010).

${ }^{5}$ J. U. Lee, D. Yonn, H. Kim et al., "Thermal conductivity of suspended pristine graphene measured by Raman spectroscopy," Phys. Rev. B 83, 081419 (2011).

${ }^{6}$ S. Chen, A. L. Moore, R. S. Ruoff et al., "Raman measurements of thermal transport in suspended monolayer graphene of variable sizes in vacuum and gaseous environments," ACS Nano 5(1), 321-328 (2011).

${ }^{7} \mathrm{~W}$. Cai et al., "Thermal transport in suspended and supported monolayer graphene grown by chemical vapor deposition," Nano Lett. 10, 1645-1651 (2010).

${ }^{8}$ Q. Y. Li, X. Zhang, and Y. D. Hu, "Laser flash Raman spectroscopy method for thermophysical characterization of 2D nanomaterials," Thermochim. Acta 592, 67-72 (2014).

${ }^{9}$ J. H. Seol, I. Jo, A. L. Moore et al., "Two-dimensional phonon transport in supported graphene," Science 328, 213-216 (2010).

${ }^{10} \mathrm{X}$. Xu et al., "Phonon transport in suspended single layer graphene," preprint at arXiv:org/abs/1012.2937 (abstract) and arXiv:org/pdf/1012.2937.pdf (full paper) (2010).

${ }^{11} \mathrm{X}$. Xu et al., "Length-dependent thermal conductivity in suspended singlelayer graphene," Nat. Commun. 5, 3689 (2014).

${ }^{12}$ Z. Wang, R. Xie, and C. T. Bui, "Thermal transport in suspended and supported few-layer graphene," Nano Lett. 11, 113-118 (2011).

${ }^{13}$ M. T. Pettes, I. Jo, Z. Yao et al., "Influence of polymeric residue on the thermal conductivity of suspended bilayer graphene," Nano Lett. 11, 1195-1200 (2011).

${ }^{14} \mathrm{H}$. Xie, L. Chen, W. Yu et al., "Temperature dependent thermal conductivity of a free-standing graphene nanoribbon," Appl. Phys. Lett. 102, 111911 (2013).

${ }^{15}$ M. H. Bae, Z. Li, Z. Aksamija et al., "Ballistic to diffusive crossover of heat flow in graphene ribbons," Nat. Commun. 4, 1734 (2013).

${ }^{16} \mathrm{E}$. Munoz, J. X. Lu, and B. I. Yakobson, "Ballistic thermal conductance of graphene ribbons," Nano Lett. 10, 1652 (2010).

${ }^{17} \mathrm{X}$. Zhang et al., "Thermal and electrical conductivity of a suspended platinum nanofilm," Appl. Phys. Lett. 86, 171912 (2005).

${ }^{18}$ M. Fujii, X. Zhang, H. Q. Xie, H. Ago, K. Takahashi, and T. Ikuta, "Measuring the thermal conductivity of a single carbon nanotube," Phys. Rev. Lett. 95, 065502 (2005).

${ }^{19}$ C. M. Orofeo, H. Hibino, K. Kawahara, Y. Ogawa, M. Tsuji, K.-i. Ikeda, S. Mizuno, and H. Ago, "Influence of Cu metal on the domain structure and carrier mobility in single-layer graphene," Carbon 50(6), 2189-2196 (2012).

${ }^{20}$ H. Ago, Y. Ito, I. Tanaka, S. Mizuno, and M. Tsuji, "Graphene sheet and method for producing the same," US Patent 8,697,230 B2 (2014).

${ }^{21}$ Z. Chen, W. Jang, W. Bao, C. N. Lau, and C. Dames, "Thermal contact resistance between graphene and silicon dioxide," Appl. Phys. Lett. 95, 161910 (2009).

${ }^{22}$ Y. K. Koh, M. H. Bae, D. G. Cahill, and E. Pop, "Heat conduction across monolayer and few-layer graphenes," Nano Lett. 10, 4363-4368 (2010).

${ }^{23}$ N. Mingo and D. A. Broido, "Carbon nanotube ballistic thermal conductance and its limits,” Phys. Rev. Lett. 95, 096105 (2005). 